\title{
BMJ Open Health research priorities for wildland firefighters: a modified Delphi study with stakeholder interviews
}

\author{
Chelsea Pelletier (D , ${ }^{1}$ Christopher Ross, ${ }^{1}$ Katherine Bailey, ${ }^{1}$ Trina M Fyfe, ${ }^{2}$ \\ Katie Cornish, ${ }^{3}$ Erica Koopmans ${ }^{3}$
}

To cite: Pelletier C, Ross C, Bailey K, et al. Health research priorities for wildland firefighters: a modified Delphi study with stakeholder interviews. BMJ Open 2022;12:e051227. doi:10.1136/ bmjopen-2021-051227

- Prepublication history and additional supplemental material for this paper are available online. To view these files, please visit the journal online (http://dx.doi.org/10.1136/ bmjopen-2021-051227).

Received 13 March 2021 Accepted 07 January 2022

Check for updates

(C) Author(s) (or their employer(s)) 2022. Re-use permitted under CC BY-NC. No commercial re-use. See rights and permissions. Published by BMJ.

${ }^{1}$ School of Health Sciences, University of Northern British Columbia, Prince George, British Columbia, Canada

${ }^{2}$ Northern Medical Program, University of Northern British Columbia, Prince George, British Columbia, Canada

${ }^{3}$ Health Research Institute, University of Northern British Columbia, Prince George, British Columbia, Canada

Correspondence to Dr Chelsea Pelletier; chelsea.pelletier@unbc.ca

\section{ABSTRACT}

Objectives The increase in global wildland fire activity has accelerated the urgency to understand health risks associated with wildland fire suppression. The aim of this project was to identify occupational health research priorities for wildland firefighters and related personnel. Design In order to identify, rank and rate health research priorities, we followed a modified Delphi approach. Data collection involved a two-stage online survey followed by semi-structured interviews.

Setting British Columbia, Canada.

Participants Participants included any current or past wildland firefighter or individuals engaged in related roles. There were 132 respondents to the first survey. Responses to the first survey were analysed to produce 10 research topics which were ranked by 75 participants in the second survey (response rate: $84 \%$ ).

Primary and secondary outcome measures The primary outcome was the identification, ranking and level of agreement of research priorities through a two-round online survey. We contextualised these findings through deductive and inductive qualitative content analysis of semi-structured interviews.

Results The most important research priorities identified were (\% consensus): effects of smoke inhalation on respiratory health $(89 \%)$, fatigue and sleep $(80 \%)$, mental health $(78 \%)$, stress $(76 \%)$ and long-term risk of disease (67\%). Interviews were completed with 14 individuals. Two main themes were developed from an inductive content analysis of interview transcripts: (1) understanding the dynamic risk environment; and (2) organisational fit of mitigation strategies.

Conclusions Participants expressed a general concern with the unknown mental and physical health impacts of their jobs, including the long-term risk of morbidity and mortality. Future research must address knowledge gaps in our understanding of the health impacts of wildland fire and work to develop appropriate mitigation strategies while considering the needs of workers and unpredictable workplace environment.

Trial registration number Open Science Framework, https://osf.io/ugz4s/

\section{INTRODUCTION}

Precipitated by climate change, accelerating wildland fire activity has extended fire seasons, increased demand for personnel

\section{Strengths and limitations of this study}

Semi-structured interviews provide contextualisation of ranked research priorities for worker health.

- Most participants were wildland firefighters, with a smaller number of people engaged in related roles.

- Limited international and national participation findings are primarily relevant to British Columbia Canada.

Data collection during the fire season means participants were actively engaged in wildland fire-related tasks.

and amplified the need to understand health impacts of wildland fire smoke for the public and those engaged in suppression efforts. ${ }^{12}$ Although there is variation across jurisdictions, wildland fire suppression typically includes a crew of frontline wildland firefighters working on a fireline, with support from related personnel such as air support (eg, water bombers), logistical coordination and operational management (eg, incident management teams). The role of a wildland firefighter is distinct from structural firefighting based on differences in smoke exposure, work structure and schedule, physical demands and other occupational hazards. ${ }^{3} \mathrm{~A}$ unique research focus, approach and priorities are needed for wildland firefighters.

Wildland firefighters are exposed to health hazards from inhalation of known air pollutants (eg, particular matter, carbon monoxide and polycyclic hydrocarbons) at levels near or above occupational exposure limits. ${ }^{4-6}$ Despite accelerating global wildland fire activity, knowledge on the health risks from occupational exposure to wildland fire is broad, inconsistent and insufficient to draw conclusions on health outcomes. ${ }^{78}$ To date, the majority of research on the health of wildland firefighters is focused on exploring acute effects of smoke exposure across a single shift or season, with most studies finding a 
reduction in lung function ${ }^{9-12}$ and increased systemic inflammation. ${ }^{13}{ }^{14}$ Modelling exposure data, Navarro et $a l^{15}$ estimated an increased lung cancer and cardiovascular disease risk among wildland firefighters, although this finding has yet to be confirmed in a prospective or longitudinal trial. Cross-sectional studies have identified associations between career length and occurrence of cardiovascular disease ${ }^{16}$ and between wildland fire smoke exposure, oxidative stress and vascular function. ${ }^{17}$ Outside of the hazards from exposure to wildland fire smoke, wildland firefighters are exposed to occupational stressors due to the nature of the profession (eg, unpredictable extended deployments, trauma from seeing homes or communities burned) and workplace culture (eg, masculine dominance). Studies focusing on mental health have identified an increased risk of post-traumatic stress among wildland firefighters and increased suicide risk. ${ }^{18}{ }^{19}$ Fireline deployments have been associated with fatigue resulting from poor sleep quality and quantity. ${ }^{20}$ Injuries are also commonly reported following work as a wildland firefighter, related to fire complexity and seasonal timing. ${ }^{21}{ }^{22}$ Few studies have been published evaluating strategies to reduce health risk. The broad focus of these intervention strategies include prescribed drinking interventions to support better thermal regulation, ${ }^{2324}$ mask wearing to reduce exposure to particulate matter ${ }^{25} 26$ and enhanced skin hygiene (eg, showering, laundering clothing) to reduce polycyclic aromatic hydrocarbon exposure. ${ }^{26}$

Health research priority setting is an important component of participatory research approaches, can maximise investments in research for funding organisations and governments and help focus research efforts across broad fields of study. ${ }^{28}$ Through research priority setting, people who stand to benefit the most from research, known as stakeholders or knowledge users, can contribute to the research process and direct research activities. ${ }^{27}$ In relation to wildland fire, stakeholder voices include frontline firefighters, people working in roles related to or supporting direct fire suppression, occupational health and safety policymakers and researchers. Health research priority setting must be well defined in scope, inclusive with broad representation, relevant to decision makers and consider the specific research context. ${ }^{27-29}$

Research priorities have been established to advance wildland fire suppression knowledge $\mathrm{e}^{30}$ and for understanding the general public health impacts of wildland fire smoke. ${ }^{31}$ We are not aware of any stakeholder-identified health research priorities related to wildland firefighters or other personnel involved in wildland fire suppression. Health research priorities are needed to ensure a coordinated and effective research plan, direct research funding by organisations and governments, support the development of appropriate mitigation strategies and ultimately improve health and well-being for all workers engaged in wildland fire suppression. The purpose of this project was to identify health research priorities for wildland firefighters and related personnel.

\section{METHODS}

This study is part of a larger project to establish health research funding priorities for the British Columbia Wildfire Service (BCWS) based on gaps in literature and stakeholder priorities. The protocol for this project has been previously published. ${ }^{32}$ To align with public health guidelines on physical distancing during the COVID-19 pandemic, we did not conduct meetings with stakeholders to discuss research priorities as indicated in our original protocol. Instead, we conducted virtual semi-structured individual interviews with a sample of survey respondents to contextualise identified research priorities, and enable broad representation given COVID-19 public health guidelines on travel and the realities of the wildland fire season.

The Delphi method is a process to gather stakeholder knowledge and experience and is commonly used to identify occupational health research priorities. ${ }^{33-36}$ The Delphi process allows for the collection of opinions from a variety of stakeholders followed by the presentation of ideas back to participants in iterative 'rounds' until consensus is reached. ${ }^{37}$ For this study, we have followed a modified Delphi method involving a two-stage online survey (SurveyMonkey) with follow-up semi-structured interviews. We modified a traditional Delphi approach by combining qualitative and quantitative data and by not having an expert panel meeting to achieve consensus following the multiple round survey. Instead, we used a modified Delphi approach to obtain a ranked list of research priorities by inviting a broad range of stakeholder participants (eg, people with lived experience as experts). Based on similar work to establish research priorities using a modified Delphi approach, we established a priori to conduct two survey rounds 3334 and determined consensus was achieved when at least $70 \%$ of respondents indicated the research priority was 'very important' or 'extremely important' during the secondround survey. ${ }^{39}$

Participants were eligible to participate if they were current or past employees of BCWS (as frontline wildland firefighters or in officer/managerial roles), researchers or trainees with an interest in wildland fire or employed in occupational health and safety. Both surveys included a basic demographic questionnaire querying age, gender and role as it related to wildland fire. Interview participants were asked to identify their job role, but we did not collect further demographic information (eg, age, gender) to protect participant confidentiality. Given the relatively small sample pool, limited number of women working for the BCWS and sensitive nature of the project in relation to the participants' workplace, the identity of some participants may be easy to deduce based on their role and was thus not collected or reported. At the end of each survey, participants could enter a draw to win one of five \$C50 Amazon gift cards. All interview participants were offered a $\$ \mathrm{C} 10$ gift card to a food or retail vendor of their choice. 


\section{Round 1: identifying research priorities}

An invitation to participate in the first survey was circulated in March 2020 via email to BCWS employees, occupational health policy makers (identified through our BCWS partners) and researchers who had recently published in the field (eg, contact information available on websites or as corresponding author). Participants were asked to list up to 10 research priorities of concern for wildland firefighters and up to 10 research priorities for related personnel in two separate lists (see online supplemental file 1). We conducted an inductive qualitative content analysis of responses. ${ }^{40-42}$ Three members of the research team read and re-read open-field responses to identify codes and key concepts provided by participants. Codes were then grouped into categories sharing common features. Discussion was used to reach consensus on final research topics. The two job categories, wildland firefighters and related personnel, were collapsed for the second survey because the topics identified by participants were similar.

\section{Round 2: rating and ranking research priorities}

The second survey was sent in June 2020 to participants who completed the first survey and expressed interest in continuing their participation. In the second survey, respondents were presented with the research topics identified in survey 1 and asked to indicate the importance of each topic on a modified 5-point Likert scale ranging from "not at all important" to "extremely important" (see online supplemental file 2). To prevent bias resulting from question order, the order of research topics was randomised for each survey respondent.

To determine the relative importance of each research topic, respondents were presented with all research topics and asked to rank them from most important to least important. To calculate the weighted average, each research topic in the data set was multiplied by the rankweight assigned by each participant before the final means were calculated. For example, a weight of 10 would be given to the respondents' highest priority, 9 to the second highest priority and so on. This was repeated for each respondent's ranked research priorities. The mean weighted average was calculated for each research topic. Participant responses were separated by job category (eg, wildland firefighter, aviation crew) to determine if there was any difference in identified research priorities based on role.

\section{Round 3: semi-structured interviews}

Semi-structured interviews allowed the opportunity for participants to provide a nuanced description of identified research topics and to identify specific projects within each umbrella topic. Eligible interview participants were at least 18 years of age and had completed both surveys. We aimed for diversity in our sample based on length of time working in a wildland fire-related role and across different job categories (eg, researchers, wildland firefighter).
Interviews were conducted by two research team members over Zoom videoconference or telephone. One researcher took detailed field notes and the other facilitated the interview using a semi-structured interview schedule. Interviews were between $22-49 \mathrm{~min}$ in length (average: $34 \mathrm{~min}$ ). The interview schedule (see online supplemental file 3) included open-ended questions about work-related health concerns, potential mitigation strategies and research questions or specific projects for each of the top five ranked research priorities.

Interviews were transcribed verbatim by a study team member. Field notes were used during analysis to provide additional contextual information. Analysis was conducted in two phases using a qualitative content analysis $^{40}{ }^{41}$ by two team members. First, we took a deductive approach to provide examples and nuanced description of each of the research topics identified in the survey. A structured categorisation matrix was created for the top five research topics based on ranking and consensus. The interview transcripts were coded for correspondence with each research topic (category); only data aligning to the matrix (eg, matched to a category or research topic) was chosen for the deductive phase of the analysis. ${ }^{41}$ Data within each category was examined to determine if any subcategories were necessary. ${ }^{42}$ Items not explicitly aligned to one of the pre-identified research topics were analysed using an inductive approach to identify additional themes. ${ }^{43}$ Inductive open coding was completed to ensure we comprehensively represented content communicated by participants and to understand issues discussed by participants not directly related to the pre-identified research topics. ${ }^{40} 41$

\section{Patient and public involvement}

Patients were not involved in this study.

\section{RESULTS}

\section{Participants}

Survey 1 was completed by 132 participants (92 men and 40 women; table 1 ). We are unable to calculate a response rate for the first survey because the invitation to participate was primarily circulated through a BCWS mailing list. Survey 2 was sent to 89 potential participants and completed by 75 respondents (response rate: $84 \%$, 48 men and 27 women). Interviews were conducted with 14 participants: 7 current or prior wildland firefighters, 3 researchers or trainees and 4 people with administrative or officer roles.

\section{Research priorities}

More than 900 research areas were suggested from participants in survey 1 which were organised into 10 categories or research topics ranked by participants in survey 2. Consensus was achieved on 5 of the 10 research topics (figure 1). Understanding and mitigating effects of smoke inhalation on respiratory health ( $89 \%$ consensus), fatigue and sleep (80\% consensus) and mental health (78\% consensus) were 
Table 1 Participant demographics

\begin{tabular}{|c|c|c|c|}
\hline & Survey $1(n=132)$ & Survey $2(n=75)$ & Interviews $(n=14)$ \\
\hline \multicolumn{4}{|l|}{ Gender (n, \%) } \\
\hline Female & 40,30 & 27,36 & $\mathrm{~N} / \mathrm{A}$ \\
\hline Male & 92,70 & 48,64 & $N / A$ \\
\hline \multicolumn{4}{|l|}{ Age (n, \%) } \\
\hline 20-29 & 45,34 & 25,33 & $\mathrm{~N} / \mathrm{A}$ \\
\hline 30-39 & 41,31 & 23,31 & $\mathrm{~N} / \mathrm{A}$ \\
\hline $40-49$ & 17,13 & 7,9 & $\mathrm{~N} / \mathrm{A}$ \\
\hline $50-59$ & 18,14 & 12,16 & $N / A$ \\
\hline $60+$ & 7,5 & 4,5 & $\mathrm{~N} / \mathrm{A}$ \\
\hline No response & 4,3 & 4,5 & $\mathrm{~N} / \mathrm{A}$ \\
\hline \multicolumn{4}{|l|}{ Role in last firefighting season $(n, \%)$} \\
\hline Aviation (eg, pilot, air attack officer) & 2,2 & 1,1 & 0 \\
\hline BCWS PWCC/fire centre staff & 25,19 & 13,17 & 2 \\
\hline BCWS zone staff & 23,17 & 14,19 & 1 \\
\hline Non-BCWS role (eg, researcher) & 16,12 & 9,12 & 4 \\
\hline Other BCWS role & 18,14 & 6,8 & 0 \\
\hline Wildland firefighter & 48,36 & 32,43 & 7 \\
\hline
\end{tabular}

BCWS, British Columbia Wildfire Service; PWCC, Provincial Wildfire Coordination Centre.

the research topics with the greatest consensus. While consensus was not reached across the entire cohort of respondents regarding the priority of long-term risk and prevalence of disease other than respiratory (67\% consensus across entire sample), within the subset of wildland firefighters and aviators, there was $81 \%$ consensus on the priority of the topic. Additionally, for the research topic camp conditions, there was consensus $(74 \%)$ within the subset of zone and control staff.

When looking at the weighted rank of each research priority, the pattern was consistent with the research priorities achieving consensus, with two notable differences. Work structure and organisational culture was the fifth topic to satisfy the consensus requirement of a rating greater than $70 \%$; however, it had the sixth highest weighted

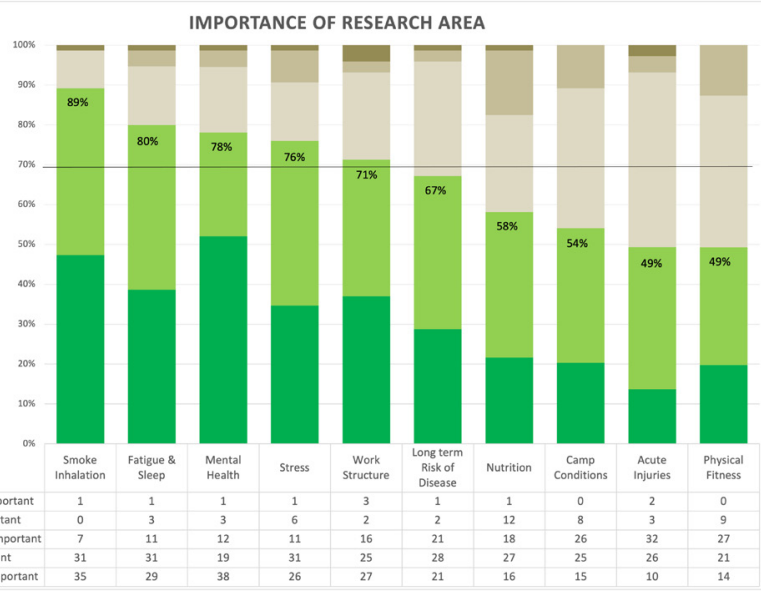

Figure 1 Consensus rating of research topics. average. Long-term risk and prevalence of disease other than respiratory was ranked fourth by participants when considering the weighted average; however, it did not reach consensus (67\%; table 2). The role of a respondent did not appear to influence the ranking of research priorities. Four of the top five areas of research as identified through the weighted average (Understanding and mitigating effects of smoke inhalation on respiratory health, fatigue and sleep, mental health and stress) also have consensus ratings greater than $70 \%$; therefore, any further research in these four areas would be addressing priority areas as identified by stakeholders.

\section{Interview findings}

In general, interview participants agreed with the ranked list of research priorities. Based on a deductive analysis, we generated areas of focus (subcategories) for three of the top five-ranked research topics (table 3). For some research topics, no subcategories were developed.

From the inductive interview analysis, we developed two overarching principles as themes relevant to every research topic:

Understanding the dynamic risk environment

Participants described the importance of understanding health risk and exposure across different roles. For example, people who work in office environments do not face the same exposures:

the office stuff or the staff that are there, I don't think they have the same concerns around smoke exposure and physiological toxins. (P8, researcher) 
Table 2 Comparison of consensus rating versus weighted average

\begin{tabular}{|c|c|c|c|c|}
\hline Research priorities & Consensus rating & Consensus rank & Weighted average & Rank \\
\hline $\begin{array}{l}\text { Understanding and mitigating effects of smoke } \\
\text { inhalation on respiratory health }\end{array}$ & $89 \%$ & $1 \mathrm{st}$ & 7.70 & $1 s t$ \\
\hline Mental health & $78 \%$ & 3rd & 6.68 & 2 nd \\
\hline Stress & $76 \%$ & 4th & 6.18 & 5th \\
\hline Nutrition, diet and hydration & $58 \%$ & 7th & 4.59 & 7 th \\
\hline Fire camp conditions & $54 \%$ & 8th & 4.11 & 8th \\
\hline Acute injuries and conditions & $49 \%$ & 9th & 4.10 & 9th \\
\hline
\end{tabular}

This different exposure may translate to different health research priorities. For instance, auxiliary staff who are typically students hired during the summer wildland fire season (May to August) may not be as concerned with long-term health risk when compared with people who work in wildland fire-related roles for their entire career:

if we're looking at an older population of firefighters, whether that be more the career wildland firefighters, I think the health priorities, the health shifts a bit. Um, in that, I'd be more concerned about a mixture of cardio-respiratory factors. Um, whether or not long-term exposure to wildland smoke might be related to later in life-like, um, uh, like lung pathologies or you know, if there's any risk factors for cardiovascular disease. (P6, researcher)

It is also important to understand and explore risks across multiple seasons because the unpredictability in fire activity may contribute to different health risks:

We think of those big fire seasons, 2017, 2018, even 2015, and like the work component is very fatiguing, but um, it's also very engaging. And then we have slower seasons like this one [2020] and we may not think of it as harder on our mental health, because we're not doing as much. But I do think it actually creates different challenges for people. So, I think that's something that we need to be aware of - even though we're not on fires, there are stressors associated with the job and it might actually be harder for people because they [do not] have that active engagement in what we see as really fulfilling work. (P4, wildland firefighter)

It really depends on the year. Because some, uh, some seasons they're so busy, and some seasons they're so slow. There's going to be like different, there's different things people care about in each, kind of different, when things are busy. (P1, wildland firefighter)
Finally, the unpredictable work structure and schedule imposes barriers and challenges to conducting research:

I mean our unpredictable work schedule could be tough for research, but the way you guys have been sending out like the surveys that we do on our own time has been awesome. Like, if we can just sit down and just take $20 \mathrm{~min}$, then by all means. But if you need to like come into our workplace and try to organize that research is a bit tougher. (P3, wildland firefighter)

\section{Organisational fit of mitigation strategies}

Participants emphasised any mitigation strategy be worker-centred, reflect worker priorities and needs, and be developed with employees. This was reflected in participants discussing that management may not always have a feel for what it is like to work on the front-line and any mitigation or management strategy not aligning with organisational realities would have minimal uptake.

people are so resistant to like any change, any, and I think it's a part of that whole disparity between management - like this is something else management's pushing down, oh they have no concept, like just ignore it...like that whole dynamic of like, just like, people sitting in office, and us like grinding out there. (P5, wildland firefighter)

Relatedly, participants discussed how organisational culture and resistance to change could present a barrier to implementing mitigation strategies:

the biggest challenge I come across is like the 'this is the way we do things' kind of attitude. (P8, researcher)

And then the organizational culture about, sort of a can-do organization and you know, we can, we get a 
Table 3 Top five ranked research topics and areas of focus identified by interview participants through deductive analysis

\section{Research topic (category)}

Understanding and mitigating effects of smoke inhalation on respiratory health

\section{Areas of focus} (subcategory)

Development of appropriate mitigation strategies

\section{Examples of meaning unit (quotes)}

"it's demoralizing sometimes being out there and not having a mask when you see, [like] ... Police officers, or other agencies that are assisting us with different things, like not even really in the smoke, and wearing like large masks to protect their health, so. Um, I think it's definitely valuable to continue looking into that and to continue pushing to get us some, um, sort of lung protection. Cause, you, you [sic] definitely feel it., Like after fire season your lung capacity is, um, it's, it's [sic] not what it was at the beginning of the season" (P1, wildland firefighter)

"I think most firefighters are pretty aware that it's not good to be breathing in what we're breathing in, but we're just at this standstill of what it seems like finding the right PPE that's appropriate for the job. So, I think research sort of on that more practical side on what, like what apparatus is going to work for us would be really important at this stage" (P4, wildland firefighter)

"You know, actual things other than like bring a mask, like if wearing mask is the only way, great. But also, beyond wearing a mask, which I think would take a while to find the right mask and the right buy in, what are the real facts, don't do it? It's like if you're doing it, what's the distances, like a lot of information about, people will be around smoke[sic], probably not wearing masks at some point. What are ways to mitigate, or like mitigate some of those things in alternate ways?" (P5, wildland firefighter)

Understand exposure "like the smoke inhalation, like what does that look like? Like what particulates, how much, like exposure, which kind of smoke you're putting in, like how, what factors influence that, like what you're actually doing and how can you like minimize that" (P5, wildland firefighter)

"the obvious one that stands out to me, that like, you know, I notice a lot more on the line, is that sort of long-term exposure piece to smoke. Um, it would probably be like fine particulate matter. Cause, there's a lot of times where, you know, in the later stages of a fire we are looking for smoke and looking for heat, and on our hand and knees in very, very [sic] fine ash with no PPE and um, and I think that probably proposes like a significant health risk. Um, I know it's been documented in other fields that kind of any really fine particulate matter like that is detrimental to your lungs, and we just go in with zero PPE and zero information about it" (P14, wildland firefighter)

\begin{tabular}{|c|c|c|}
\hline \multirow[t]{4}{*}{ Fatigue and sleep } & \multirow[t]{2}{*}{$\begin{array}{l}\text { Determine optimal } \\
\text { amounts of sleep }\end{array}$} & $\begin{array}{l}\text { "what is the optimal amount, amount of sleep that like a firefighter should be } \\
\text { getting? Or what is the optimal amount of rest in order to fully, like, kind of } \\
\text { reset our minds and reset our bodies, uh to get ready for that next fourteen-day } \\
\text { deployment. I don't know, like fourteen to three just seems like it was pulled out } \\
\text { of the air, so, um, l'd be curious to know like what, what is the proper amount of } \\
\text { rest that we should be getting" (P1, wildland firefighter) }\end{array}$ \\
\hline & & $\begin{array}{l}\text { "the quality of sleep, the length of shifts and whether or not they're able to } \\
\text { achieve-or obtain-quality sleep while they're in the field, and whether or not } \\
\text { that is, um, impacting on their safety while working" (P6, researcher) }\end{array}$ \\
\hline & $\begin{array}{l}\text { Understand impacts } \\
\text { of long-term fatigue }\end{array}$ & $\begin{array}{l}\text { "the short-term fatigue of like day-to-day, as opposed to the long-term fatigue of } \\
\text { a season and how that affects you season to season kind of thing" (P3, wildland } \\
\text { firefighter) }\end{array}$ \\
\hline & & $\begin{array}{l}\text { "when you have, have } 8 \text { hours off between shifts, including you know going } \\
\text { home, cooking a meal, taking a shower, all things that you do at home, so that } \\
\text { adds up over time. So yeah, I think it'd be interesting to see how that would } \\
\text { affect um kind of like overall fatigue, long term fatigue" (P2, wildland firefighter) }\end{array}$ \\
\hline
\end{tabular}


Table 3 Continued

\begin{tabular}{lll}
\hline $\begin{array}{l}\text { Research topic } \\
\text { (category) }\end{array}$ & $\begin{array}{l}\text { Areas of focus } \\
\text { (subcategory) }\end{array}$ & Examples of meaning unit (quotes) \\
\hline Mental health & $\begin{array}{l}\text { Understand mental } \\
\text { health impacts } \\
\text { during and after fire } \\
\text { season }\end{array}$ & $\begin{array}{l}\text { "people kind of just starting out, that you know, everything seems like super, } \\
\text { sulity of what you went through kind of sinks in" (P1, wildland firefighter) } \\
\text { realing }\end{array}$ \\
& &
\end{tabular}

"but you know, the day-to-day and how that effects morale at your base, or how that might affect behaviour dynamics between groups of people when you're stuck out there at a miserable fire for weeks at a time, that should be something that we talk about more" (P10, wildland firefighter/office role)

Optimise supports "Important to have information on what we're getting ourselves into and the and strategies to long-term effects of this job" (P4, wildland firefighter)

increase awareness

"The stigma and not really recognizing some of the effects of, you know, exposure and post-traumatic stress" (P6, researcher)

"Some education in that area to people in the field would be good. Cause I think a lot of folks are not you know, aware of the mental toll that this job might have... it's certainly not something that's discussed as a standard as part of the training" (P10, wildland firefighter/office role)

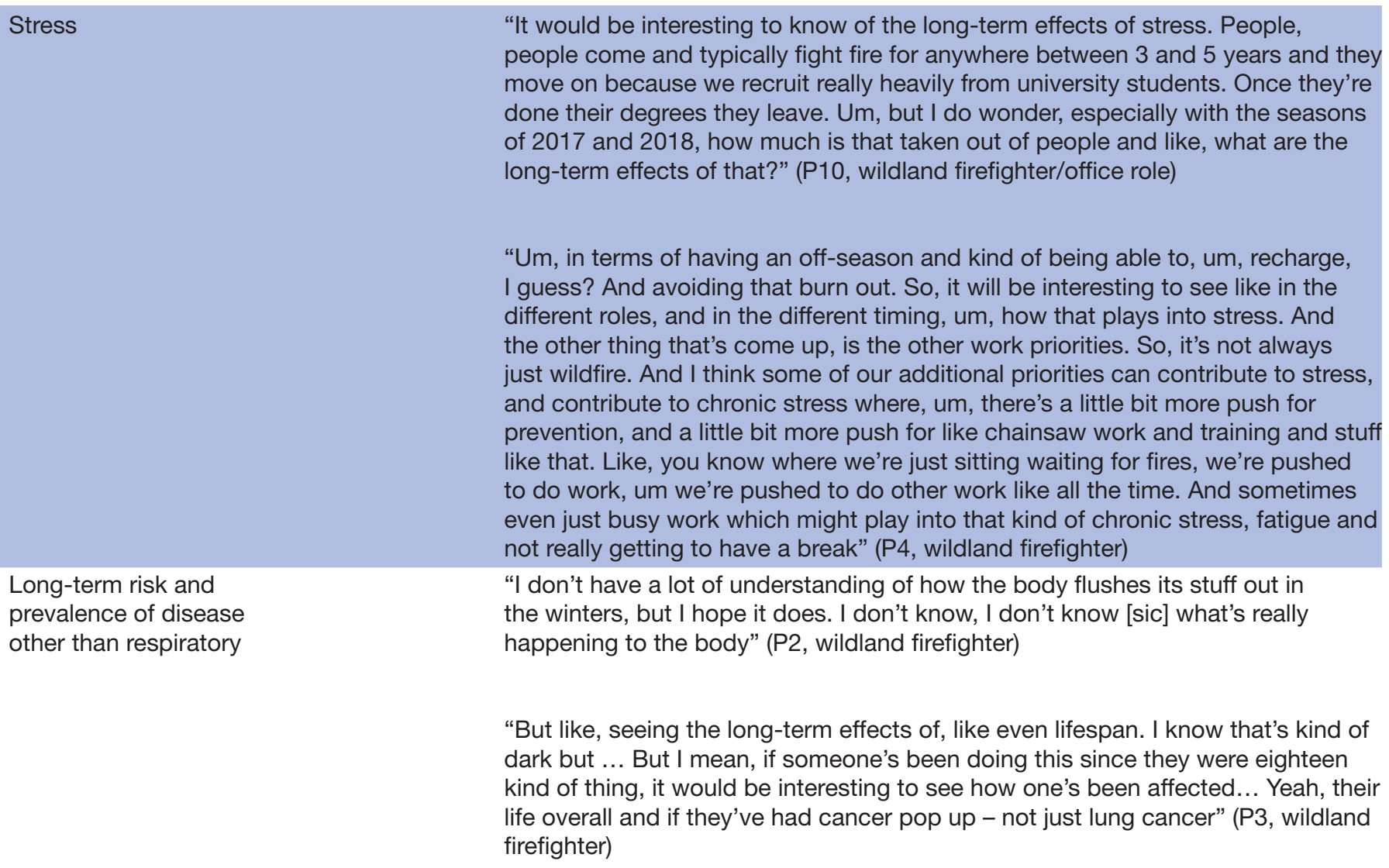

PPE, personal protective equipment.

lot done-yes-we can take care of things, and this is the way we've always done it. And uh, yeah, it's certainly an organization, I think. The-yeah-so just sort of pushing through that organizational culture. (P13, administrative/office role)

\section{DISCUSSION}

Following best practice guidelines for research priority setting, ${ }^{27}$ the aim of this project was to identify health research priorities for wildland firefighters and related personnel. Five research topics achieved consensus across 
the entire sample: understanding and mitigating effects of smoke inhalation on respiratory health, fatigue and sleep, mental health, stress and work structure and organisational culture. A sixth research topic, long-term risk and prevalence of disease other than respiratory, nearly reached consensus $(67 \%$ agreement) and was ranked highly by all participants. Based on interviews with relevant stakeholders, we have developed specific areas of study within each research topic. We identified two themes common to all research topics: (1) understanding the dynamic risk environment across wildland fire seasons and roles and (2) organisational fit of mitigation strategies. Despite a different risk environment, the health concerns identified by participants in the current study parallel those by structural firefighters, ${ }^{44}$ although there is considerably less knowledge specific to health outcomes associated with wildland fire exposure and substantial gaps in knowledge on the longterm health impacts. ${ }^{8}$

The most important research topic identified by participants was related to smoke exposure and respiratory health, with $89 \%$ of participants indicating it was extremely or very important. During interviews, participants focused on the need for a better understanding of the exposures and health risks associated with wildland fire smoke and the desire for feasible, effective personal protective equipment to protect against smoke inhalation. While many participants mentioned masks, they also noted the difficulties and practicality of wearing a mask in the field while fighting fire for long hours. Two recent papers have explored mask wearing to protect against wildland fire smoke, suggesting they may be effective at reducing particulate matter exposure even if used for only part of a shift; however, neither study reported any data on user experience. ${ }^{25}{ }^{26}$ Ultimately, participants expressed the desire to be engaged in the development of any mitigation strategy and corresponding occupational health policy as essential to ensure uptake. Collaborative intervention development can help researchers better understand the wildland fire occupational context, ensure alignment with organisation policy and support effective implementation-a process recently used to develop interventions to address psychosocial health and physical fitness of wildland firefighters in Ontario, Canada. ${ }^{45}$

Participants indicated a need to understand the cumulative mental and physical health toll of both busy and quiet fire seasons, and recovery in the off-season. Of particular concern was the need to understand the risk of chronic fatigue resulting from an increasing length of fire seasons and reduced opportunity to recover. The nature of the occupation often necessitates sleeping at a fire camp, where wildland firefighters do not typically achieve appropriate quality and quantity of sleep. ${ }^{20} 46$ While research has documented poor sleep conditions and reduced cognitive function in-field, ${ }^{20} 4647$ research questions remain about the optimal work to rest schedule for wildland firefighting, strategies for recovery in the offseason and how to balance sleep hygiene with the realities of wildland fire operations and crew management.
Notably, while the research topic long-term risk and prevalence of disease other than respiratory did not reach consensus among the entire sample, it was ranked highly and reached consensus $(81 \%)$ among people involved in direct fire suppression. Designing and conducting research to understand the cumulative impacts of multiple seasons of wildland fire exposure is a considerable challenge and the long-term health impacts of wildland firefighting are poorly understood, ${ }^{78}$ although we do note ongoing work by the US National Institute for Occupational Safety and Health. ${ }^{48}$ In British Columbia, many front-line wildland firefighters are post-secondary students hired as auxiliary employees during the summer (May to August) wildland fire season. The transient and mobile workforce creates considerable challenges to evaluating risk related to morbidity and mortality outcomes over multiple seasons. Similarly, the unpredictability of a given fire season means generating consistent evidence or measuring changes in health outcomes across any one season is challenging due to the variable exposure. For example, some participants mentioned unique challenges during less active fire seasons such as a different mental health toll from not being able to engage in work they enjoy and find meaningful. Thus, while some fire seasons may be 'quieter' in terms of fire activity and respiratory health risk (eg, lower smoke exposure), they may impose a different mental health risk. While our research priorities can be used to support researchers to make decisions on prioritising collective research efforts, findings also suggest the need for sustainable, flexible research infrastructure to adapt and understand variable risk environments.

\section{Limitations}

The email invitation to participate was sent in March 2020. This was done to ensure data collection was not impacted by the busiest point in the fire season (typically June to August in British Columbia). Because of this, many auxiliary/seasonal staff had not yet started work and may be under-represented in our sample. Participants were asked to list their top 10 research priorities for wildland firefighters and related personnel separately in the first survey. Based on very similar research topics identified for both job categories and suggestions by participants in the open field question, we collapsed the two job categories for the stage 2 survey. This may have reduced our ability to tease out specific research topics for related personnel and identified research priorities are likely more applicable to wildland firefighters. Occupational health policymakers and researchers were identified by convenience sampling through our partnership with BCWS and by extracting contact information from recently published papers in the field, which may have limited the breadth of stakeholder participant perspectives.

\section{CONCLUSIONS}

This project represents the first attempt to develop a comprehensive set of research priorities for wildland 
firefighters and related personnel. Our process included a broad range of stakeholders, from frontline staff to those working in occupational health and safety policy, with national and international representation. Stakeholders consistently identified the need for research to understand the physical and mental health risks of wildland firefighting, and how this risk may lead to long-term morbidity and mortality across multiple fire seasons or an entire career. Appropriate, feasible mitigation, prevention and/or management strategies are urgently needed to address health concerns for workers directly or indirectly engaged in wildland fire suppression.

Contributors CP lead study conception and design, data collection, analysis and manuscript writing, and is the guarantor of this work. CR was involved in the development, distribution and analysis of online surveys, and assisted in preparation of the manuscript. KB supported interview data collection, transcription, interview analysis and preparation of the manuscript. TMF, EK and KC provided critical feedback on data collection, study design, assisted with survey and interview analysis. All authors participated in the interpretation of the results, provided critical revision of and approved the final manuscript.

Funding Funding for this project was provided by the British Columbia Ministry of Forests, Lands, Natural Resource Operations and Rural Development, British Columbia Wildfire Service, through its membership with the Canadian Partnership for Wildland Fire Science.

\section{Competing interests None declared.}

Patient consent for publication Not applicable.

Ethics approval This study involves human participants and was approved by University of Northern British Columbia Research Ethics Board (E2019.1220.076). Participants gave informed consent to participate in the study before taking part.

Provenance and peer review Not commissioned; externally peer reviewed.

Data availability statement No data are available. Due to the nature of this research and to protect participant confidentiality, no additional data from this study are available.

Supplemental material This content has been supplied by the author(s). It has not been vetted by BMJ Publishing Group Limited (BMJ) and may not have been peer-reviewed. Any opinions or recommendations discussed are solely those of the author(s) and are not endorsed by BMJ. BMJ disclaims all liability and responsibility arising from any reliance placed on the content. Where the content includes any translated material, BMJ does not warrant the accuracy and reliability of the translations (including but not limited to local regulations, clinical guidelines, terminology, drug names and drug dosages), and is not responsible for any error and/or omissions arising from translation and adaptation or otherwise.

Open access This is an open access article distributed in accordance with the Creative Commons Attribution Non Commercial (CC BY-NC 4.0) license, which permits others to distribute, remix, adapt, build upon this work non-commercially, and license their derivative works on different terms, provided the original work is properly cited, appropriate credit is given, any changes made indicated, and the use is non-commercial. See: http://creativecommons.org/licenses/by-nc/4.0/.

ORCID iD

Chelsea Pelletier http://orcid.org/0000-0002-8009-8014

\section{REFERENCES}

1 Pechony O, Shindell DT. Driving forces of global wildfires over the past millennium and the forthcoming century. Proc Natl Acad Sci U S A 2010;107:19167-70.

2 Westerling AL. Increasing Western us forest wildfire activity: sensitivity to changes in the timing of spring. Philos Trans $R$ Soc Lond B Biol Sci 2016;371:20150178.

3 Booze TF, Reinhardt TE, Quiring SJ, et al. A screening-level assessment of the health risks of chronic smoke exposure for wildland firefighters. J Occup Environ Hyg 2004;1:296-305.

4 Navarro KM, Cisneros R, Noth EM, et al. Occupational exposure to polycyclic aromatic hydrocarbon of wildland firefighters at prescribed and wildland fires. Environ Sci Technol 2017;51:6461-9.
5 Miranda Al, Martins V, Cascão P, et al. Wildland smoke exposure values and exhaled breath indicators in firefighters. $J$ Toxicol Environ Health A 2012;75:831-43.

6 Materna BL, Jones JR, Sutton PM, et al. Occupational exposures in California wildland fire fighting. Am Ind Hyg Assoc J 1992;53:69-76.

7 Groot E, Caturay A, Khan Y, et al. A systematic review of the health impacts of occupational exposure to wildland fires. Int J Occup Med Environ Health 2019;32:121-40.

8 Koopmans E, Cornish K, Fyfe TM, et al. Health risks and mitigation strategies from occupational exposure to wildland fire: a scoping review. J Occup Med Toxicol 2022;17:2.

9 Gaughan DM, Cox-Ganser JM, Enright PL, et al. Acute upper and lower respiratory effects in wildland firefighters. $J$ Occup Environ Med 2008;50:1019-28.

10 Jacquin L, Michelet P, Brocq F-X, et al. Short-Term Spirometric changes in wildland firefighters. Am J Ind Med 2011;54:819-25.

11 Rothman N, Ford DP, Baser ME, et al. Pulmonary function and respiratory symptoms in wildland firefighters. J Occup Med 1991;33:1163-7.

12 Liu D, Tager IB, Balmes JR, et al. The effect of smoke inhalation on lung function and airway responsiveness in wildland fire fighters. Am Rev Respir Dis 1992;146:1469-73.

13 Gianniou N, Giannakopoulou C, Dima E, et al. Acute effects of smoke exposure on airway and systemic inflammation in forest firefighters. $J$ Asthma Allergy 2018;11:81-8.

14 Main LC, Wolkow AP, Tait JL, et al. Firefighter's Acute Inflammatory Response to Wildfire Suppression. J Occup Environ Med 2020;62:145-8.

15 Navarro KM, Kleinman MT, Mackay CE, et al. Wildland firefighter smoke exposure and risk of lung cancer and cardiovascular disease mortality. Environ Res 2019;173:462-8.

16 Semmens EO, Domitrovich J, Conway K, et al. A cross-sectional survey of occupational history as a wildland firefighter and health. Am J Ind Med 2016;59:330-5.

17 Gaughan DM, Siegel PD, Hughes MD, et al. Arterial stiffness, oxidative stress, and smoke exposure in wildland firefighters. Am J Ind Med 2014;57:748-56.

18 McFarlane AC. Relationship between psychiatric impairment and a natural disaster: the role of distress. Psychol Med 1988;18:129-39.

19 Stanley IH, Hom MA, Gai AR, et al. Wildland firefighters and suicide risk: examining the role of social disconnectedness. Psychiatry Res 2018;266:269-74.

20 Jeklin AT, Davies HW, Bredin SSD, et al. Fatigue and sleep patterns among Canadian wildland firefighters during a 17-day fire line deployment. J Occup Environ Hyg 2020;17:364-71.

21 Britton C, Lynch CF, Ramirez M, et al. Epidemiology of injuries to wildland firefighters. Am J Emerg Med 2013;31:339-45.

22 Moody VJ, Purchio TJ, Palmer CG. Descriptive analysis of injuries and illnesses self-reported by wildland firefighters. Int $J$ Wildland Fire 2019;28:412-9.

23 Raines J, Snow R, Petersen A, et al. Pre-shift fluid intake: effect on physiology, work and drinking during emergency wildfire fighting. Appl Ergon 2012;43:532-40.

24 Raines J, Snow R, Petersen A, et al. The effect of prescribed fluid consumption on physiology and work behavior of wildfire fighters. Appl Ergon 2013;44:404-13.

25 De Vos AJBM, Cook A, Devine B, et al. Effect of protective filters on fire fighter respiratory health during simulated bushfire smoke exposure. Am J Ind Med 2006;49:740-50.

26 Cherry N, Galarneau J-M, Kinniburgh D, et al. Exposure and absorption of PAHs in wildland firefighters: a field study with pilot interventions. Ann Work Expo Health 2021;65:148-61.

27 Sibbald SL, Singer PA, Upshur R, et al. Priority setting: what constitutes success? A conceptual framework for successful priority setting. BMC Health Serv Res 2009;9:43.

28 Viergever RF, Olifson S, Ghaffar A, et al. A checklist for health research priority setting: nine common themes of good practice. Health Res Policy Sys 2010;8:36.

29 Priority setting for health research: lessons from developing countries. The Working group on priority setting. Health Policy Plan 2000;15:130-6.

30 Sankey S. Blueprint for wildland fire science in Canada (2019-2029). Natural Resources Canada, 2018. Available: https://www.nrcan.gc. $\mathrm{ca} /$ our-natural-resources/forests-forestry/wildland-fires-insectsdisturban/blueprint-wildland-fire-science-canada-2019-2029/21614 [Accessed 12 Mar 2021]

31 Errett NA, Roop HA, Pendergrast C, et al. Building a practicebased research agenda for Wildfire smoke and health: a report of the 2018 Washington Wildfire smoke risk communication Stakeholder synthesis symposium. Int J Environ Res Public Health 2019;16:2398. 
32 Koopmans E, Fyfe T, Eadie M, et al. Exploring prevention and mitigation strategies to reduce the health impacts of occupational exposure to wildfires for wildland firefighters and related personnel: protocol of a scoping study. Syst Rev 2020;9:119.

33 Okoli C, Pawlowski SD. The Delphi method as a research tool: an example, design considerations and applications. Inf Manage 2004;42:15-29.

34 lavicoli S, Rondinone B, Marinaccio A, et al. Identification of research priorities in occupational health. Occup Environ Med 2005;62:71-2.

35 Lalloo D, Demou E, Smedley J, et al. Current research priorities for UK occupational physicians and occupational health researchers: a modified Delphi study. Occup Environ Med 2018;75:830-6.

36 Jones J, Hunter D. Qualitative research: consensus methods for medical and health services research. BMJ 1995;311:376-80.

37 Hasson F, Keeney S, McKenna H. Research guidelines for the Delphi survey technique. J Adv Nurs 2000;32:1008-15.

38 Gagliardi D, Rondinone BM, Mirabile M, et al. The perspective of European researchers of national occupational safety and health Institutes for contributing to a European research agenda: a modified Delphi study. BMJ Open 2017;7:e015336.

39 Kouyoumdjian FG, Schuler A, Mclsaac KE, et al. Using a Delphi process to define priorities for prison health research in Canada. BMJ Open 2016:6:e010125.

40 Elo S, Kyngäs $\mathrm{H}$. The qualitative content analysis process. $J \mathrm{Adv}$ Nurs 2008;62:107-15.
41 Hsieh H-F, Shannon SE. Three approaches to qualitative content analysis. Qual Health Res 2005;15:1277-88.

42 Bengtsson $M$. How to plan and perform a qualitative study using content analysis. NursingPlus Open 2016;2:8-14.

43 Sandström B, Willman A, Svensson B, et al. Perceptions of national guidelines and their (non) implementation in mental healthcare: a deductive and inductive content analysis. Implement Sci 2015;10:43.

44 Jahnke SA, Poston WSC, Jitnarin N, et al. Health concerns of the U.S. fire service: perspectives from the firehouse. Am J Health Promot 2012;27:111-8.

45 Leduc C, Giga SI, Fletcher IJ, et al. Participatory development process of two human dimension intervention programs to foster physical fitness and psychological health and well-being in wildland firefighting. Int J Environ Res Public Health 2021;18:7118.

46 Vincent GE, Aisbett B, Hall SJ, et al. Fighting fire and fatigue: sleep quantity and quality during multi-day wildfire suppression. Ergonomics 2016;59:932-40.

47 McGillis Z, Dorman SC, Robertson A, et al. Sleep quantity and quality of Ontario wildland firefighters across a low-hazard fire season. $J$ Occup Environ Med 2017;59:1188-96.

48 The National Institute for Occupational Safety and Health (NIOSH). Wildland firefighter exposure and health effects study, 2020. Available: https://www.cdc.gov/niosh/topics/firefighting/ wffhealthstudy.html [Accessed 21 Oct 2021] 